\title{
Treatment of Patients with Prediabetes in a Primary Care Setting 2011-2018: an Observational Study
}

\author{
Sidra L. Speaker, BA ${ }^{7}$, Radhika Rastogi, BA ${ }^{7}$, Tamara A. Sussman, $M D^{2}$, Bo Hu, $P h D^{3}$, \\ Anita D. Misra-Hebert, MD, $\mathrm{MPH}^{3,4}$, and Michael B. Rothberg, MD, $\mathrm{MPH}^{1,4}$
}

${ }^{1}$ Cleveland Clinic Lerner College of Medicine, Case Western Reserve University, Cleveland, OH, USA; ${ }^{2}$ Taussig Cancer Institute, Cleveland Clinic, Cleveland, OH, USA; ${ }^{3}$ Quantitative Health Services, Cleveland Clinic, Cleveland, OH, USA; ${ }^{4}$ Center for Value-Based Care Research, Cleveland Clinic, Cleveland, $\mathrm{OH}$, USA.

\begin{abstract}
BACKGROUND: Over one third of American adults are at high risk for developing diabetes, which can be delayed or prevented using interventions such as medical nutrition therapy (MNT) or metformin. Physicians' self-reported rates of prediabetes treatment are improving, but patterns of actual referral, prescription, and MNT visits are unknown.
\end{abstract}

OBJECTIVE: To characterize treatment of prediabetes in primary care.

DESIGN: We conducted a retrospective cohort study using electronic health record data. We described patterns of treatment and used multivariable logistic regression to evaluate the association of patient factors and $\mathrm{PCP}$-specific treatment rate with patient treatment.

PATIENTS: We included overweight or obese outpatients who had a first prediabetes-range hemoglobin Alc (HbAlc) during 2011-2018 and had primary care provider (PCP) follow-up within a year.

MAIN MEASURES: We collected patient characteristics and the following treatments: metformin prescription; referral to MNT, diabetes education, endocrinology, or bariatric medicine; and MNT visit. We did not capture withinvisit physician counseling.

KEY RESULTS: Of 16,713 outpatients with prediabetes, $20.4 \%$ received treatment, including metformin prescriptions (7.8\%) and MNT referrals (11.3\%), but only $7.4 \%$ of referred patients completed a MNT visit. The strongest predictor of treatment was the patient's PCP's treatment rate. Some PCPs never treated prediabetes, but two treated more than half of their patients; $62 \%$ had no patients complete a MNT visit. Being younger or female and having higher body mass index or HbAlc were also positively associated with treatment. Compared to white patients, black patients were more likely to receive MNT referral and less likely to receive metformin.

CONCLUSIONS: Almost $80 \%$ of patients with new prediabetes never received treatment, and those who did receive referrals had very poor visit completion. Treatment rates appear to reflect provider rather than patient preferences.

Prior Presentations An earlier version of this work was presented at the 2018 National Annual Meeting for the Society for General Internal Medicine, April 11-14, 2018.

Received April 8, 2020

Accepted November 18, 2020

Published online January 15, 2021
J Gen Intern Med 36(4):923-9

DOI: $10.1007 /$ s11606-020-06354-4

(C) Society of General Internal Medicine 2021

\section{INTRODUCTION}

Over one third of American adults have prediabetes, ${ }^{1}$ which conveys high risk for progression to diabetes ${ }^{2,3}$ and increases all-cause mortality. ${ }^{4}$ Clinical guidelines recommend low-risk, low-cost interventions including medical nutrition therapy (MNT), exercise, and metformin in order to delay or prevent progression to diabetes, but clinical uptake is uncertain. ${ }^{5-7}$

Since 2014, the US Preventive Task Force (USPTF) has recommended intervention for prediabetes, and longitudinal measurement of MNT use is needed to estimate implementation. $^{8,9}$ In a recent survey of physicians, $67 \%$ reported recommending MNT to at least some patients, ${ }^{10}$ and national visit-level data indicate that diet is discussed at less than a fifth of visits and metformin is prescribed at less than $3 \%{ }^{11}$ Together, these findings suggest that even providers who report treating prediabetes do not do so consistently. Similarly, a longitudinal examination of metformin for prediabetes found that although $90 \%$ of providers reported prescribing, only $10 \%$ of patients received it. ${ }^{10}$ Longitudinal measures of MNT referral are lacking.

Second, existing visit-level measures of diet intervention do not discriminate between office-based counseling and MNT referral. Office counseling should not replace MNT or the multidisciplinary Diabetes Prevention Program (DPP). An American Diabetes Association (ADA) Consensus Report recommends that all overweight or obese patients with prediabetes be referred to intensive lifestyle intervention such as a DPP or MNT with a nutritionist or registered dietitian. ${ }^{12}$

Finally, cross-sectional surveys cannot capture trends in prediabetes treatment, identify treatment rates at the physician level, or differentiate referrals from completed visits. To overcome these limitations and present a better understanding of trends in prediabetes treatment, we examined metformin prescriptions and MNT referrals within a large health system from 2011 to 2018. We aimed to describe treatment patterns and patient factors associated with treatment and to understand the relationship between referrals for MNT and completed visits. 


\section{RESEARCH DESIGN AND METHODS}

\section{Study Population}

This retrospective cohort study was approved by the Cleveland Clinic Institutional Review Board. We included Cleveland Clinic primary care patients with a newly identified prediabetes-range (5.70-6.49\%) hemoglobin A1c (HbA1c) recorded January 2011 through December 2018, and a primary care provider (PCP) visit within 12 months following the initial HbAlc. ${ }^{7}$ As the largest provider of primary care in Northeast Ohio, the Cleveland Clinic serves a representative subdivision of the general local patients (Table S1). We excluded patients with type I or preexisting type II diabetes (a diabetes diagnosis or diabetes-range $(>6.49 \%$ ) $\mathrm{HbAlc}$ prior to the first $\mathrm{HbA1c}$ ), known prediabetes (diagnosis preceding the first prediabetes-range $\mathrm{HbAlc}$ by $>1$ year), or confounding disease processes (e.g., gestational hyperglycemia and steroid-related hyperglycemia). To limit our population to patients for whom dietary intervention is indicated, we excluded patients with a $\mathrm{BMI}<25$. 5,12

The PCP field in our electronic health record (EHR) is sometimes inaccurate or empty. We therefore assigned PCP based on the plurality of primary care visits within the year following the patient's first prediabetes-range HbAlc, with ties attributed to the PCP field. We excluded patients whose PCP had $<30$ patients in the sample in order to allow for clustering by PCP in a multilevel model.

\section{Variables}

All data were extracted from the EHR and processed in the R statistical software (Version 3.6.1) using R Studio. ${ }^{13,14} \mathrm{We}$ collected the following: demographics, body mass index (BMI), current smoking status, prediabetes- and diabetesrelated diagnoses, and $\mathrm{HbA} 1 \mathrm{c}$. Because there was no numeric code for prediabetes prior to international disease classification (ICD)-10, we used diagnosis names entered into the EHR during the period covered by ICD-9 (Supplement 2). Subsequently, we used the prediabetes code R73.03.

As HbA1c values increased, we reclassified patients as they progressed to "prediabetes" and then "diabetes." We did not allow for regression of stages if subsequent $\mathrm{HbA} 1 \mathrm{c}$ values improved. We considered any of the following to indicate progression to diabetes: $\mathrm{HbA} 1 \mathrm{c} \geq 6.5 \%,{ }^{2}$ ICD code for diabetes, or outpatient insulin prescription for $>28$ days. We did not use diagnosis of "prediabetes" or related ICD codes to define progression to prediabetes, because use of these codes is heterogeneous and most often not associated with a HbAlc. Although glucose tolerance testing and fasting glucose can identify prediabetes, ${ }^{15}$ fasting status and glucose tolerance test context are not consistently identifiable in our EHR. Therefore, we used only $\mathrm{HbA} 1 \mathrm{c}$ results to identify prediabetes.

\section{Outcome Measures}

Our primary outcome was any attempt at treating prediabetes, including a prescription for metformin or referral to any of the following: MNT, diabetes education, endocrinology consultation, and bariatric medicine consultation. We considered MNT from 2 perspectives. Referrals represent the physician's intention to treat, while visits identify actual treatment. We did not collect information regarding office-based counseling because such counseling is variably documented, and few physicians have the time or expertise to counsel effectively, which is why the ADA recommends that overweight or obese patients with prediabetes receive referral to MNT or a DPP. ${ }^{12}$ Patients were censored once they developed diabetes, so treatment beginning after that point was not counted. Time to treatment was the interval between the first PCP visit after prediabetes onset and referral or prescription.

\section{Statistical Analysis}

We described the study population and treatment frequencies as means with standard deviations for continuous variables and proportions for categorical variables. We assessed differences between treatment groups using chi-square and ANOVA. Unadjusted cumulative probabilities of treatment were plotted over time since the first PCP visit after the initial prediabetes-range $\mathrm{HbA1c}$.

To compare PCP intervention rates, we modeled the intervention probability of individual PCPs using a multilevel model, and then standardized those probabilities to incorporate the patient characteristics of each PCP's panel, as described below. We used fixed effects regression coefficients with $95 \%$ confidence intervals to explore factors associated with treatment.

For the multilevel logistic regression model, there were fixed effects for patient demographic and clinical variables and a random effect for PCP. For each patient, we input the PCP identity and the patient's demographic and clinical data to back-predict treatment probability. Means and standard deviations for patient panel treatment rates provided initial estimates for PCP-specific intervention rates. We multiplied initial estimates by PCP panel standardization factors, which were generated for each PCP by dividing the whole-group mean treatment rate by PCP panel-specific mean treatment probability, which we imputed using patient variables only in a whole-sample logistic regression model. Because the effect of patient variables was standardized across the sample, intervention rate estimates should reflect only the PCP-related propensity to treat, rather than patient panel characteristics. Finally, PCP-specific panel-adjusted mean intervention rates and $95 \%$ confidence intervals were compared against the overall mean PCP intervention rate in order to identify highand low-intervening PCPs. We used the same approach to compare use of MNT and metformin separately.

We regressed visit completion on patient factors as above, using a subset of 878 patients who were referred to MNT and whose PCP $(n=50)$ referred at least 10 patients to MNT, at least one of whom completed a visit. PCP tertile for MNT visit completion rate was included as a covariate in this model. 
Finally, to understand the contribution of each model factor to predicting the outcome, we calculated the area under the receiver operating characteristic (ROC) curve omitting one predictor at a time. To limit the effect of possible overfitting, we split the full cohort randomly into half training and half testing datasets. The predictor that produced the largest reduction in the area under the curve was considered to offer the greatest contribution to the decision to treat. ${ }^{16,17}$

\section{RESULTS}

Of 46,710 patients with a new prediabetes-range HbAlc during the study period, 16,713 patients met criteria for inclusion (Figure S1). Of these, $57 \%$ received a diagnostic code for prediabetes, and $20.4 \%$ received any treatment modality, including metformin prescription or referral to endocrinology, bariatric care, diabetes education, or MNT over a follow-up duration of up to 8 years; $5.8 \%$ were prescribed metformin only, $9.3 \%$ received a MNT referral only, and $2.0 \%$ received both. Incidence of treatment was highest at the initial PCP visit following a prediabetes-range $\mathrm{HbA} 1 \mathrm{c}$, with $2.7 \%$ of patients receiving an MNT referral and $2.8 \%$ receiving a metformin prescription within 4 weeks (Fig. 1). After 1 year of follow-up, $4.5 \%$ of patients were prescribed metformin and $5.7 \%$ were referred to MNT. Cumulative incidence of each treatment increased over time. Of patients with the maximum followup time of 8 years, over one quarter were treated, while $11.8 \%$ progressed to diabetes.

\section{Factors Associated with Treatment}

Patients who received a prediabetes diagnostic code were more likely to receive treatment than those who remained undiagnosed $(23.9 \%$ vs. $10.8 \%, p<0.001)$. Other clinical factors associated with MNT referral or metformin prescription appear in Table 1. There was a steady increase in diagnosis of new prediabetes from $42.2 \%$ in 2011 to $66.7 \%$ in 2018; however, rates of all types of treatment and rates of visit completion did not change during this period (Fig. 2).

There was little correlation between PCP prescribing of metformin and MNT referral $\left(r^{2}=.04\right)$, and no correlation between a PCP's MNT referral rate and the proportion of their patients who completed a MNT visit $\left(r^{2}<.0001\right)$ (Figure S2). Therefore, we created separate multivariable models for each treatment (i.e., MNT referral and metformin). In both models, the strongest predictor of an individual receiving treatment was their PCP's overall treatment rate (Table S2). Female sex, longer follow-up, younger age, higher initial HbAlc, and higher BMI were also independently associated with receiving treatment (Table 2). Black race was positively associated with MNT referral and negatively associated with metformin prescription, while being a current smoker was negatively associated with MNT referral.

Of $1881(11.3 \%)$ patients who received MNT referrals, $140(7.4 \%)$ completed at least one visit. Thus, less than $1 \%$ of patients with prediabetes visited a nutritionist. MNT referral follow-through rates were lower than follow-through rates for other referrals, including endocrinology ( $15.4 \%$ of 162 referrals), diabetes education ( $24.4 \%$ of 193 referrals), and bariatric consultation (24.6\% of 544 referrals). In multivariable analysis, PCP tertile for visit follow-through was again the strongest predictor of completing a MNT visit. Younger age and longer duration of prediabetes were also associated with visit completion (Table 3).

\section{PCP Prescribing Behavior}

Among 245 PCPs, unadjusted treatment rates ranged from 0 to $58 \%$, with only 2 PCPs treating at least half their patients. Two physicians never treated prediabetes, 18 (7.3\%) never prescribed metformin, $15(6.1 \%)$ never referred to MNT, and

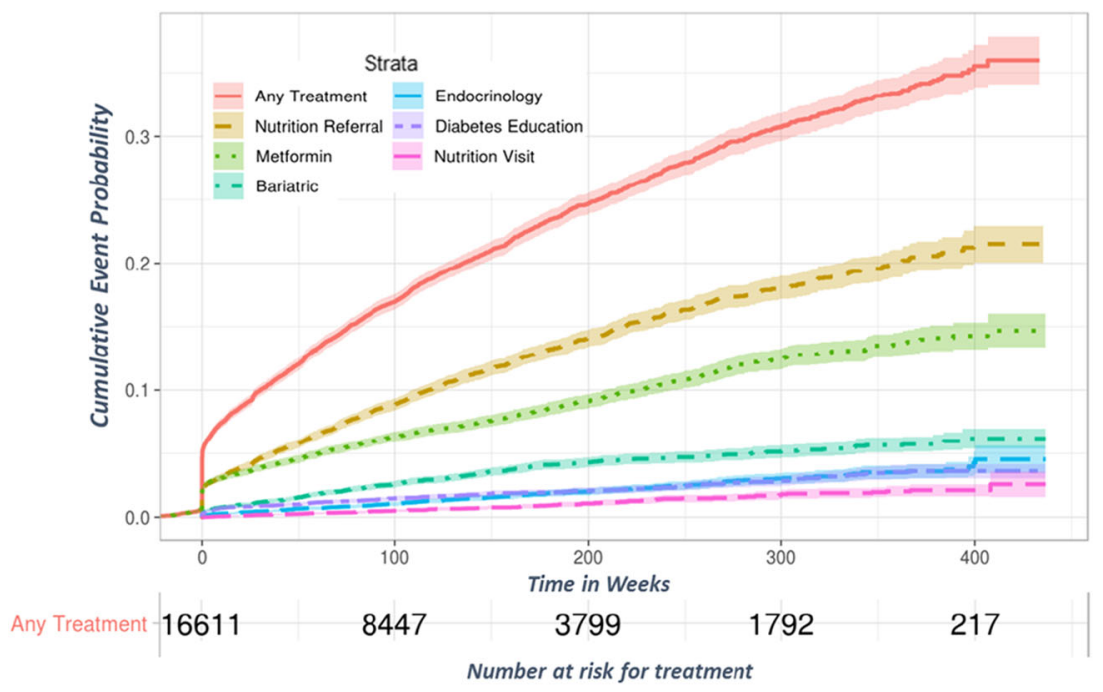

Figure 1 Cumulative incidence of various treatment modalities in patients with prediabetes between the years 2011 and 2018. 
Table 1 Treatment with Diet Referral and/or Metformin Prescription Within 1 Year from PCP Visit After Prediabetes Diagnosis During 20112018

\begin{tabular}{|c|c|c|c|c|c|}
\hline & None & Referral & Metformin & Both & $p$ \\
\hline & $13,856(82.9 \%)$ & $1548(9.3 \%)$ & $976(5.8 \%)$ & $333(2 \%)$ & \\
\hline Age (mean (SD)) & $62.3(12.8)$ & $56.8(12.1)$ & $54.7(12.1)$ & $52.8(11.4)$ & $<0.001$ \\
\hline Female $(\%)$ & $7165(51.7)$ & $1017(65.7)$ & $609(62.4)$ & $238(71.5)$ & $<0.001$ \\
\hline Race $(\%)$ & & & & & $<0.001$ \\
\hline White & $11,022(79.5)$ & $1029(66.5)$ & $753(77.2)$ & $216(64.9)$ & \\
\hline Black & $2014(14.5)$ & $427(27.6)$ & $175(17.9)$ & $103(30.9)$ & \\
\hline Other & $820(5.9)$ & $92(5.9)$ & $48(4.9)$ & $14(4.2)$ & \\
\hline BMI (mean (SD)) & $32.8(6.1)$ & $36.2(7.8)$ & $35.9(7.6)$ & $39.2(8.4)$ & $<0.001$ \\
\hline HbAlc (mean (SD)) & $5.94(0.19)$ & $5.96(0.19)$ & $6.02(0.21)$ & $6.01(0.21)$ & $<0.001$ \\
\hline Smoker $(\%)$ & $1784(12.9)$ & $156(10.1)$ & $131(13.4)$ & $38(11.4)$ & 0.012 \\
\hline Prediabetes diagnosis $(\%)$ & $9588(69.2)$ & $1310(84.6)$ & $818(83.8)$ & $303(91.0)$ & $<0.001$ \\
\hline Follow-up time (years) & $2.8(2.1)$ & $4.0(2.3)$ & $3.5(2.2)$ & $4.4(2.2)$ & $<0.001$ \\
\hline
\end{tabular}

BMI body mass index, HbAlc hemoglobin Alc

$151(62 \%)$ never had a patient complete a MNT visit. High variability among PCPs was evident for each modality. For overall treatment, 73 (30\%) PCPs had intervention rates significantly above and 104 (42\%) significantly below the mean (Fig. 3). There was even more variability in PCP rates of MNT referral (Figure S3) and metformin prescription (Figure S4).

\section{DISCUSSION}

As an asymptomatic chronic disease, prediabetes is most appropriately identified and treated in the primary care setting. More than 15 years ago, the Diabetes Prevention Program (DPP) study demonstrated that diabetes could be prevented through diet and exercise or metformin. Nevertheless, we found that fewer than 1 in 10 patients with prediabetes seen in primary care received evidence-based treatment within 1 year of diagnosis. ${ }^{5}$ Although approximately $11 \%$ eventually received a MNT referral, less than $1 \%$ of all patients ever completed a visit. Over time, PCPs increasingly diagnosed prediabetes, but this did not translate into increased treatment. Most notable was the extreme variability in PCP intervention rates, which ranged from 0 to $58 \%$, and a patient's probability of being treated was most strongly associated with their choice of physician. At the patient level, both metformin prescription and MNT referral were associated with female sex, younger age, and higher BMI. Compared to white patients, black patients were more likely to receive a MNT referral and less likely to be prescribed metformin.

Other sources confirm some of the demographic findings. Pharmacy claims data indicate that obese patients are more likely to receive metformin, while national survey results demonstrate associations between younger age, higher BMI, and black race with lifestyle intervention. ${ }^{11,18,19}$ The use of specific treatments for different groups may reflect a combination of physician and patient preferences. Thus, the relative

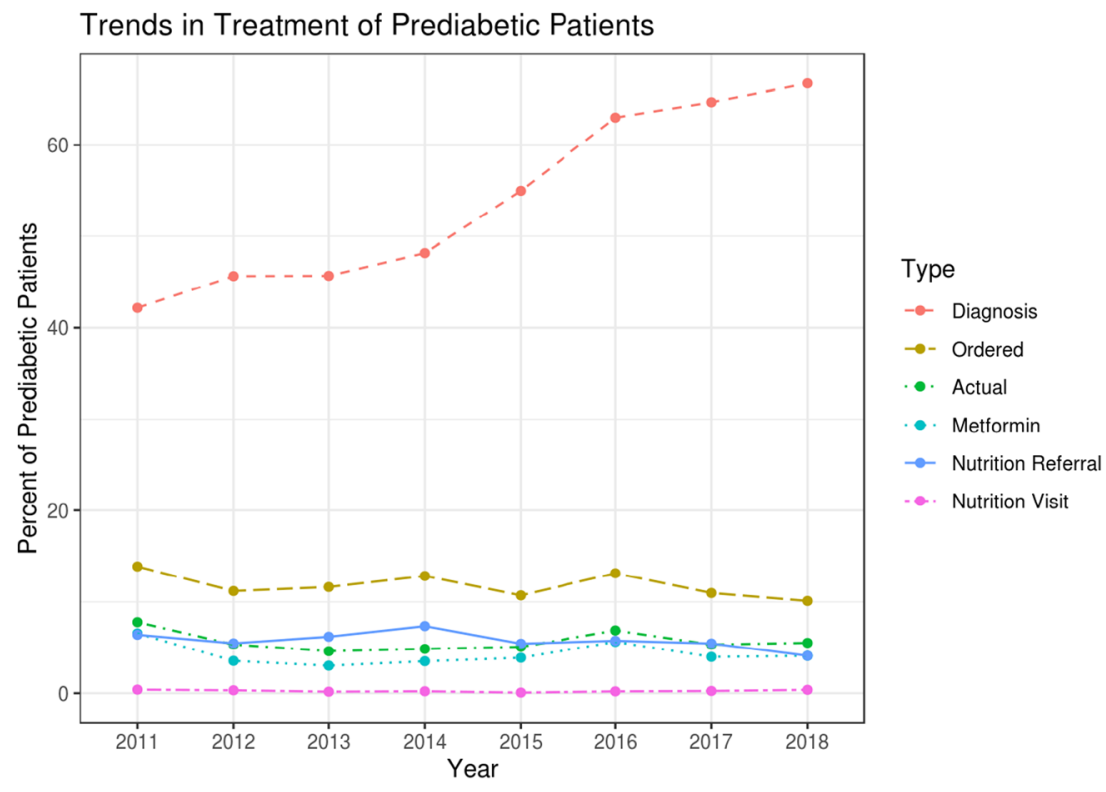

Figure 2 Trends in any treatment within 1 year of first visit after prediabetes onset (Ordered, nutrition referral placed or metformin prescribed; Actual, nutrition visit completed or metformin prescribed; Metformin, metformin prescribed). 
Table 2 Patient and Physician Factors Associated with Nutrition Referral or Metformin Prescription in a Multivariable Model

\begin{tabular}{|c|c|c|c|c|c|c|}
\hline \multirow[t]{3}{*}{ Predictor } & \multicolumn{3}{|c|}{ Nutrition referral } & \multicolumn{3}{|c|}{ Metformin prescription } \\
\hline & OR & Lower & Upper & OR & Lower & Upper \\
\hline & & \multicolumn{2}{|c|}{$(95 \% \mathrm{CI})$} & & \multicolumn{2}{|l|}{$(95 \% \mathrm{CI})$} \\
\hline Age (decades) & 0.75 & 0.72 & 0.79 & 0.62 & 0.59 & 0.66 \\
\hline Female & 1.44 & 1.29 & 1.61 & 1.47 & 1.29 & 1.67 \\
\hline BMI (per 1 unit) & 1.06 & 1.05 & 1.07 & 1.04 & 1.03 & 1.05 \\
\hline \multicolumn{7}{|l|}{ Race } \\
\hline Black & 1.42 & 1.25 & 1.61 & 0.79 & 0.67 & 0.92 \\
\hline Other & 1.02 & 0.81 & 1.28 & 0.71 & 0.53 & 0.94 \\
\hline Smoker & 0.78 & 0.65 & 0.92 & 0.85 & 0.71 & 1.02 \\
\hline HbA1c (per $1.0 \%)$ & 1.55 & 1.19 & 2.04 & 10.50 & 7.71 & 14.20 \\
\hline Years followed & 1.22 & 1.19 & 1.25 & 1.16 & 1.13 & 1.19 \\
\hline \multicolumn{7}{|c|}{ PCP intervention rate* } \\
\hline High vs mean & 1.91 & 1.68 & 2.18 & 2.62 & 2.26 & 3.04 \\
\hline Low vs mean & 0.42 & 0.36 & 0.49 & 0.37 & 0.32 & 0.44 \\
\hline
\end{tabular}

*PCP intervention rate is the classification of the patient panel-adjusted primary care provider probability of providing intervention as higher, the same, or lower than the mean

BMI body mass index, HbAlc hemoglobin Alc

undertreatment of black patients with metformin may be due to real or perceived preferences for avoiding medication in the context of asymptomatic disease. ${ }^{20,21}$ Reduced use of metformin for older patients is supported by the DPP study, but lower rates of MNT referral are not. Undertreatment may reflect a shift in priorities away from long-term disease prevention in older patients or recognition that Medicare does not cover MNT for prediabetes. ${ }^{22}$ The strong association of metformin prescription with $\mathrm{HbAlc}$ may reflect PCPs simply treating higher prediabetes-range $\mathrm{HbAlc}$ as "almost diabetic."

National data have demonstrated variability in prediabetes treatment by region and institution. ${ }^{11}$ Our results indicate that there is even more variability among physicians within a single institution, something that appears not to have been reported before. The wide variation in adjusted treatment rates suggests that physicians are primarily responsible for the lack of treatment. Indeed, some physicians did not treat prediabetes at all, and only two physicians treated even half of their patients. Potential explanations for these low rates include

Table 3 Logistic Regression Model Using Patient and Primary Care Provider Factors to Explain Which Patients with Prediabetes Are Treated with a Dietitian Visit, Out of Patients Who Received Nutrition Referral

\begin{tabular}{llll}
\hline \hline Predictor & OR & Lower & Upper \\
\cline { 3 - 4 } & & $\mathbf{( 9 5 \%} \mathbf{C I )}$ & \\
\hline Age (decades) & 0.80 & 0.64 & 0.99 \\
Female & 1.36 & 0.81 & 2.36 \\
BMI (per 1 unit) & 0.99 & 0.96 & 1.02 \\
Race & & & \\
$\quad$ Black & 0.88 & 0.52 & 1.46 \\
Other & 1.01 & 0.33 & 2.58 \\
Smoker & 0.96 & 0.35 & 2.23 \\
HbA1c (per 1.0\%) & 1.26 & 0.36 & 4.30 \\
Years followed & 1.29 & 1.15 & 1.45 \\
PCP treatment tertile & 2.91 & 1.32 & 6.25 \\
$\quad$ High vs middle & 0.45 & 0.27 & 0.77 \\
Low vs middle & & & \\
\hline
\end{tabular}

BMI body mass index, HbAlc hemoglobin Alc limited PCP knowledge and PCP belief that prevention of diabetes is hampered by patient lack of motivation and systemic barriers. ${ }^{23}$ In addition, our system has no clinical decision support or quality metrics to promote prediabetes treatment.

Schmittdiel et al. identified multiple system- and patientlevel interventions to improve diabetes prevention. ${ }^{24}$ For example, information technology can facilitate annual $\mathrm{HbAlc}$ ordering to monitor patients with prediabetes, and lifestyle coaching can help patients lose weight. ${ }^{25,26}$ Indeed, the 2015 USPTF recommendations to screen 40-70-year-old patients for abnormal glucose as part of the cardiovascular risk assessment may have contributed to improvement in prediabetes diagnosis $^{9}$ as electronic reminders were added to prompt screening for $\mathrm{HbAlc}$. It is unclear why MNT referrals did not increase as well, especially because surveys of physicians reported increasing referrals to lifestyle change programs over this time $\left(11 \%\right.$ in $2015^{7}$ to $31 \%$ in $\left.2017^{10}\right)$. In our system, increases in diagnosis did not lead to more treatment, thus systematizing $\mathrm{HbAlc}$ ordering to identify more patients with prediabetes is unlikely to improve treatment rates. Instead, effort should focus on prompting physician responses to prediabetes-range $\mathrm{HbAl}$ c values and supporting patient follow-through.

The poor follow-through on MNT referrals is particularly concerning. Previous studies have not reported rates of visit completion. ${ }^{11}$ Physicians appear to strongly influence visit completion rates, perhaps by selecting appropriate patients, facilitating appointments, or motivating patients to attend. This finding warrants qualitative exploration to identify the determinants of high completion rates.

This lack of follow-up also suggests a potential role for improved insurance coverage, which PCPs have identified as an obstacle to diabetes prevention. ${ }^{23,27}$ Medicare only commenced coverage for diabetes prevention programs in 2018, and programs are still not widely available, including at our 


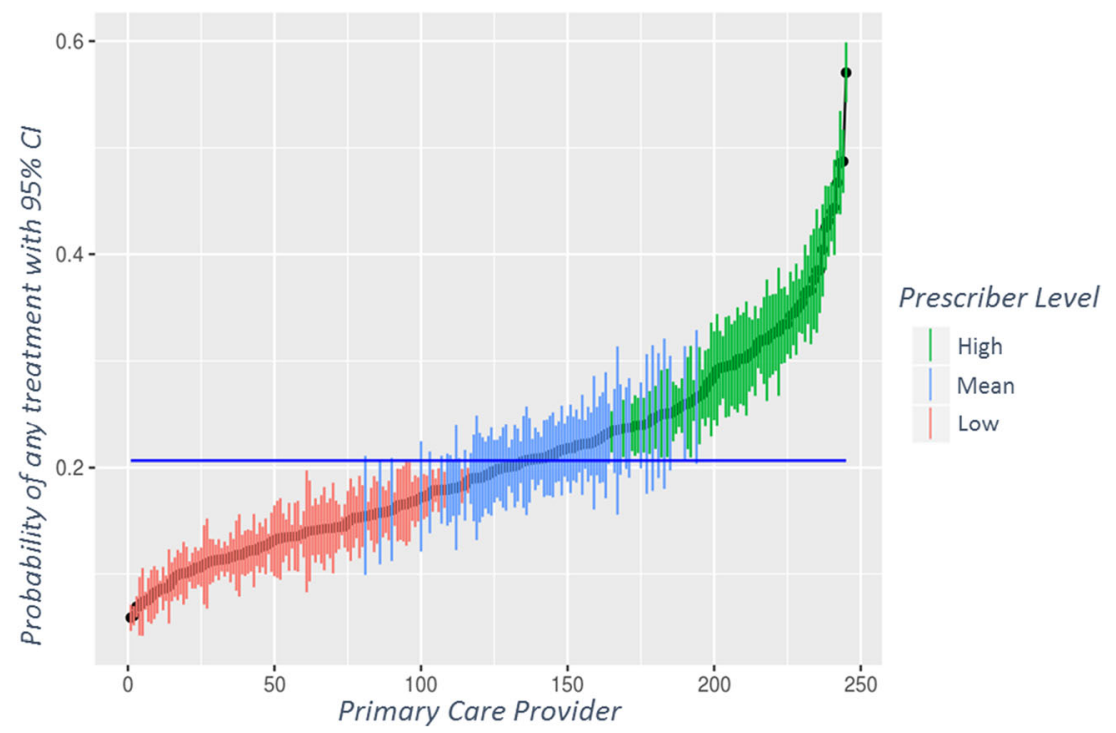

Figure 3 Adjusted probability of intervening with any treatment by individual primary care provider. The blue line is the mean intervention rate.

institution. ${ }^{28}$ Most insurers, including Medicare, do not reimburse MNT visits until a patient has documented diabetes or kidney disease, despite ample evidence of the clinical and cost-effectiveness of MNT for prediabetes. ${ }^{12,27,29,30}$ Cost issues cannot explain the low usage of metformin, which is widely available as an inexpensive generic medication. More work is needed to understand this dichotomy.

While changes to insurance coverage will take time, physician prescribing of metformin could be addressed immediately. The rarity of metformin treatment has been widely reported, with rates ranging from $<1$ to $10.3 \%$ in various patient samples. ${ }^{10,11,18,19,31}$ Whether increases can best be achieved through education, clinical decision support or quality measures are yet to be determined. As with all potential quality measures, the goal should not be $100 \%$ attainment, to leave room for patient autonomy.

\section{Limitations}

We did not capture referrals to DPPs because these services are not available within our healthcare system. While the DPP represents the best evidence-based care, it was not available in almost three quarters of counties with the highest diabetes prevalence in 2017, and efforts to improve use of existing resources in the absence of the DPP remain important. ${ }^{28} \mathrm{We}$ did not capture exercise because exercise is not routinely recorded in the EHR. We did not capture in-office counseling by PCPs; however, this should not replace a visit with a registered dietitian. ${ }^{12}$ We also did not capture income or insurance, which may contribute meaningfully to a patient's probability of completing the MNT visit. We included only referrals placed after identification of prediabetes and visits completed after a referral was placed, so some events may have been missed. Conversely, we captured all orders in the specified time frame regardless of who placed them, so some
PCPs may have had treatments ordered by others attributed to them. We expect any patient misattribution to bias PCP variability toward the null. Finally, our study was conducted in a single large health system. Results may not be generalizable to other systems.

Type 2 diabetes is a slowly developing, preventable disease, yet more than $80 \%$ of overweight or obese patients with prediabetes in this cohort never received evidence-based treatment despite follow-up in primary care. Low rates of treatment could be attributed to individual PCPs, who varied widely in their propensity to treat and to get their patients to complete a MNT visit. Qualitative study is required to identify why many PCPs continue to prescribe metformin rarely and to understand the poor patient follow-through with MNT referrals. New quality measures might incentivize treatment for prediabetes, and widespread reimbursement for MNT and healthcare-initiated MNT coordination might improve visit completion. In particular, the establishment of diabetes prevention programs within Medicare-covered entities would facilitate referral and enrollment of many patients with currently untreated prediabetes.

Corresponding Author: Michael B. Rothberg, MD, MPH; Center for Value-Based Care Research, Cleveland Clinic, Cleveland, OH, USA (e-mail: rothbem@ccf.org).

Supplementary Information The online version contains supplementary material available at https://doi.org/10.1007/s11606-02006354-4.

\section{Compliance with Ethical Standards:}

Conflict of Interest: Dr. Misra-Hebert has received grant research support from the Agency for Healthcare Research and Quality K08 HSO24128, grants from NHLBI, grants from Novo Nordisk, Inc, grants from Merck Inc, grants from Boehringer Ingelheim Pharmaceuticals, Inc, outside the submitted work. The other authors have no conflicts of interest to disclose. 


\section{REFERENCES}

1. Prevention $\mathrm{C}$ for $\mathrm{DC}$ and National diabetes statistics report, 2017. Atlanta, GA Centers Dis Control Prev US Dep Heal Hum Serv. 2017.

2. Association $\mathrm{AD}$. 2. Classification and diagnosis of diabetes: standards of medical care in diabetes-2020. Diabetes Care. 2020;43(Supplement 1):S14-S31. https://doi.org/10.2337/dc20-S002.

3. Tabák AG, Herder C, Rathmann W, Brunner EJ, Kivimäki M. Prediabetes: a high-risk state for diabetes development. Lancet. 2012;379(9833):2279-2290. https://doi.org/10.1016/S0140-6736(12) 60283-9.

4. Cai X, Zhang Y, Li M, et al. Association between prediabetes and risk of all cause mortality and cardiovascular disease: updated meta-analysis. BMJ. 2020;370:m2297. https://doi.org/10.1136/bmj.m2297.

5. Knowler WC, Barrett-Connor E, Fowler SE, et al. Reduction in the incidence of type 2 diabetes with lifestyle intervention or metformin. $\mathrm{N}$ Engl J Med 2002;346(6):393-403. https://doi.org/10.1056/ NEJMoa012512.

6. Li G, Zhang $\mathbf{P}$, Wang $\mathbf{J}$, et al. The long-term effect of lifestyle interventions to prevent diabetes in the China Da Qing Diabetes Prevention Study: a 20-year follow-up study. Lancet. 2008;371(9626):1783-1789. https://doi.org/10.1016/S0140-6736(08) 60766-7

7. Association $\mathrm{AD}$. 3. Prevention or delay of type 2 diabetes: standards of medical care in diabetes-2020. Diabetes Care. 2020;43(Supplement 1):S32-S36. https://doi.org/10.2337/dc20-S003.

8. LeFevre ML. Behavioral counseling to promote a healthful diet and physical activity for cardiovascular disease prevention in adults with cardiovascular risk factors: U.S. Preventive Services Task Force Recommendation Statement. Ann Intern Med 2014;161(8):587. https://doi. org/10.7326/M14-1796.

9. Siu AL. Screening for abnormal blood glucose and type 2 diabetes mellitus: U.S. Preventive Services Task Force Recommendation Statement. Ann Intern Med 2015;163(11):861-868. https://doi.org/10.7326/ M15-2345.

10. Keck JW, Thomas AR, Hieronymus L, Roper KL. Prediabetes knowledge, attitudes, and practices at an academic family medicine practice. J Am Board Fam Med 2019;32(4):505-512. https://doi.org/10.3122/ jabfm.2019.04.180375.

11. Wu J, Ward E, Lu ZK. Addressing lifestyle management during visits involving patients with prediabetes: NAMCS 2013-2015. J Gen Intern Med 2018:1412-1418. https://doi.org/10.1007/s11606-018-4724-z.

12. Evert AB, Dennison M, Gardner CD, et al. Nutrition therapy for adults with diabetes or prediabetes: a consensus report. Diabetes Care 2019;42(5):731-754. https://doi.org/10.2337/DCI19-0014.

13. Team RC. R: a language and environment for statistical computing. 2019

14. Wickham H. Tidyverse. 2017. https://cran.r-project.org/ package=tidyverse.

15. 2. Classification and diagnosis of diabetes: standards of medical care in diabetesd2019. Diabetes Care. 2019;42:S13-S28. https://doi.org/10. 2337/dc19-S002.

16. Kattan Mw. Judging new markers by their ability to improve predictive accuracy. JNCI J Natl Cancer Inst 2003;95(9):634-635. https://doi.org/ 10.1093/jnci/95.9.634.

17. Kattan Mw. Evaluating a new marker's predictive contribution. Clin Cancer Res 2004;10(3):822-824. https://doi.org/10.1158/1078-0432. CCR-03-0061.
18. Wu J, Ward E, Threatt T, Lu ZK. Metformin prescribing in low-income and insured patients with prediabetes. J Am Pharm Assoc (2003) 2017;57(4):483-487. https://doi.org/10.1016/j.japh.2017.04.008.

19. Moin T, Li J, Duru OK, et al. Metformin prescription for insured adults with prediabetes from 2010 to 2012: a retrospective cohort study. Ann Intern Med 2015;162(8):542-548. https://doi.org/10.7326/M14-1773.

20. Shiyanbola OO, Brown CM, Ward EC. "I did not want to take that medicine": African-Americans' reasons for diabetes medication nonadherence and perceived solutions for enhancing adherence. Patient Prefer Adherence 2018;12:409-421. https://doi.org/10.2147/PPA S152146.

21. Shenolikar RA, Balkrishnan R, Camacho FT, Whitmire JT, Anderson RT. Race and medication adherence in medicaid enrollees with type-2 diabetes. J Natl Med Assoc 2006;98(7):1071-1077.

22. Medical nutrition therapy insurance coverage. https://www.medicare. gov/coverage/nutrition-therapy-services. Accessed April 6, 2020

23. Tseng E, Greer RC, O'Rourke P, et al. National survey of primary care physicians' knowledge, practices, and perceptions of prediabetes. J Gen Intern Med 2019. https://doi.org/10.1007/s11606-019-05245-7.

24. Schmittdiel JA, Adams AS, Dlott R. Preventing diabetes in high-risk patients: time for a system-level approach to disease prevention. $\mathrm{J}$ Gen Intern Med 2019;34(8):1367-1368. https://doi.org/10.1007/s11606019-04994-9.

25. Bowen ME, Schmittdiel JA, Kullgren JT, Ackermann RT, O'Brien MJ. Building toward a population-based approach to diabetes screening and prevention for us adults. Curr Diab Rep. 2018;18(11). https://doi.org/ 10.1007/s11892-018-1090-5.

26. Schmittdiel JA, Adams SR, Goler N, et al. The impact of telephonic wellness coaching on weight loss: a "Natural Experiments for Translation in Diabetes (NEXT-D)" study. Obesity (Silver Spring) 2017;25(2):352-356. https://doi.org/10.1002/oby.21723.

27. Sun Y, You W, Almeida F, Estabrooks P, Davy B. The effectiveness and cost of lifestyle interventions including nutrition education for diabetes prevention: a systematic review and meta-analysis. J Acad Nutr Diet. 2017;117(3):404-421.e36. https://doi.org/10.1016/j.jand.2016.11.016.

28. Jayapaul-Philip B, Dai S, Kirtland K, Haslam A, Nhim K. Availability of the National Diabetes Prevention Program in United States counties, March 2017. Prev Chronic Dis. 2018;15(9). https://doi.org/10.5888/ pcd15.180063.

29. Briggs Early K, Stanley K. Position of the Academy of Nutrition and Dietetics: the role of medical nutrition therapy and registered dietitian nutritionists in the prevention and treatment of prediabetes and type 2 diabetes. J Acad Nutr Diet 2018;118(2):343-353. https://doi.org/10. 1016/j.jand.2017.11.021.

30. Raynor HA, Davidson PG, Burns H, et al. Medical nutrition therapy and weight loss questions for the evidence analysis library prevention of type 2 diabetes project: systematic reviews. J Acad Nutr Diet 2017;117(10):1578-1611. https://doi.org/10.1016/j.jand.2017.06.361.

31. Tseng E, Yeh HC, Maruthur NM. Metformin use in prediabetes among U.S. adults, 2005-2012. In: Diabetes Care. Vol 40. American Diabetes Association Inc.; 2017:887-893. https://doi.org/10.2337/dc16-1509.

Publisher's Note Springer Nature remains neutral with regard to jurisdictional claims in published maps and institutional affiliations. 